\title{
Positive Surgical Margins at Radical Prostatectomy: Importance of Intra-Operative Bladder Neck Frozen Sections
}

\author{
Kogenta Nakamura, Ali Kasraeian, Satoshi Anai, John Pendleton, Charles J. Rosser \\ Department of Urology, Gainesville, and Division of Urology, Jacksonville, University of Florida, \\ Florida, USA
}

\begin{abstract}
Objective: To determine if intraoperative frozen sections of the bladder neck during radical prostatectomy (RP) could decrease the incidence of final positive surgical margins at the bladder neck.

Materials and Methods: This prospective cohort study included 51 consecutive men who underwent anatomic RP at University of Florida \& Shands Jacksonville. All patients had intraoperative frozen section of bladder neck sent for analysis. Preoperative, operative, and postoperative data were collected and analyzed.

Main Outcome Measures: Outcome measures were intraoperative bladder neck margin status, final pathologic bladder neck margin status, and postoperative urinary complications. Median follow-up for the 51 patients was 22 months.

Results: The final positive surgical margin rate was 20\% (10 patients). An additional three patients had positive surgical margins at the bladder neck intraoperatively. These patients then had a wider resection of the affected bladder neck until the frozen sections were negative for cancer or prostatic tissue. Final pathologic evaluation of bladder neck margin was negative for tumor or persistent prostatic tissue in all 51 men.

Conclusion: With intra-operative frozen sections, we were able to obtain a negligible positive bladder neck margin rate. Surgeons who are still on the learning curve for RP should consider intra-operative frozen section of the bladder neck.
\end{abstract}

Key words: prostate cancer; prostatectomy; bladder neck; frozen sections; surgical margins

Int Braz J Urol. 2007; 33: 746-51

\section{INTRODUCTION}

Radical prostatectomy (RP) is a wellestablished treatment option for localized prostate cancer. Technical refinements introduced by Walsh $\&$ Dokker not only decrease morbidity associated with the procedure, but also are associated with overall better oncologic outcomes (1). One of the oncologic outcomes routinely assessed is surgical margins. The most frequent site of a positive margin is the apex, followed by the posterolateral, anterior, and bladder neck (2). Incompletely resected cancers (i.e., positive surgical margin or positive lymph nodes) are at increased risk of treatment failure (3). Current surgical technique leaves minimal to negligible amount of tissue that can be further resected at the apex, anterior, or posterolateral sites. However, this is not the case at the bladder neck. Following bladder neck sparing RP, an adequate amount of tissue remains that can be analyzed by intra-operative frozen sections to ensure negative surgical margins at the bladder neck. The objective of this study was to determine if intra- 
operative frozen sections of the bladder neck taken during anatomic RP could decrease the incidence of final positive surgical margins at the bladder neck. We herein report the bladder neck margin status of 51 consecutive RP patients treated at our institute.

\section{MATERIALS AND METHODS}

\section{Study Population}

Fifty-one consecutive patients with localized prostate cancer undergoing bladder neck sparing anatomic RP at University of Florida \& Shands Jacksonville from October 2003 to December 2005 were included in the study. No patients treated during this time period were excluded. All data needed for this study was collected and recorded as part of standard-of-care for these patients. In 2006, Institutional IRB approval was obtained to examine the medical records and gather pertinent information.

Pretreatment evaluations included medical history, physical examination with digital rectal examination, initial prostate specific antigen (PSA) level, and measurement and determination of Gleason score by prostate needle biopsy. Different laboratories may have been used to measure PSA of different patients. Further evaluations with bone scan or computed tomography scan were obtained according to the preference of the treating urologist. Expanded Prostate Cancer Index Composite (EPIC) questionnaires were completed preoperatively, and 6 weeks, 6 months, and 12 months postoperatively.

\section{Tumor Grade and Stage}

The 2002 Tumor-Node-Metastasis (TNM) staging system was used for clinical staging (4). Radical prostatectomy specimens were inked and processed as previously reported (5). Surgical margins $\leq 3 \mathrm{~mm}$ were considered focal and surgical margins $>3 \mathrm{~mm}$ were considered extensive. The pelvic lymph node dissection was omitted in patients with a low likelihood of lymph node involvement. After a meticulous bladder neck dissection and transection, intra-operative sections from the left, anterior, right and posterior bladder neck were sent to pathology. Briefly, 3-mm. thick transmural circumferential biopsy of the bladder neck margin was obtained along with any other suspicious tissue on the base of the bladder. Margins were inked with a marking pen. A portion of the intra-operative specimen was analyzed as a frozen section and another portion analyzed after formalin fixation. If tumor or even benign glands were evident at the intra-operative bladder neck margin, more of the bladder neck was resected until a negative margin on frozen section was obtained. In those patients with a large bladder neck defect, bladder neck reconstruction was performed as previously stated (6). Lymph nodes removed during bilateral pelvic lymph node dissection were examined either immediately by frozen section and subsequently by permanent sections, or by permanent sections only. Radical prostatectomy specimens were graded histologically according to the Gleason grading system and categorized by pathology (7).

\section{Outcome Assessment}

Hospital records were reviewed for several key outcomes. First, the intra-operative frozen section and final pathologic margin status were recorded. Next, complications and the urinary bother as assessed by EPIC questionnaire were recorded pre-operatively, 6 weeks, 6 months and 12 months post-operatively in 32 of the 51 men.

\section{Follow-up}

The patients were followed 6-8 weeks after surgery and 4-6 months thereafter using PSA monitoring and digital rectal examinations. Biochemical failure was defined as detectable serum PSA level (>0.4 ng/mL) (8). No patient developed a clinical recurrence without a biochemical recurrence. Follow-up information was collected as the patients returned for clinic visits. At the end of the study, after IRB approval, the information needed was gathered from each patient's hospital record. In a few cases, patients had moved or lived at a great distance and follow-up information was obtained by contacting the patients' physicians elsewhere or from other hospitals. Median follow-up of the study was 22 months. 


\section{RESULTS}

A total of 51 men with a mean age of 64 years (range, 45-74 years) comprised the study cohort. A description of the study population, including race, serum PSA, Gleason score of prostate biopsy, and clinical stage, is shown in Table-1. The majority of patients presented with serum PSA between 4.1-10 $\mathrm{ng} / \mathrm{mL}(57 \%)$, Gleason score $\leq 6(65 \%)$, and $\mathrm{T} 1 \mathrm{c}$ disease $(75 \%)$.

Of the 51 men, 15 (29\%) underwent a bilateral nerve sparing RP; 24 (47\%) men underwent a unilateral nerve sparing RP; and 12 (24\%) men underwent non-nerve sparing procedure. A majority of the subjects $(86 \%)$ underwent a pelvic lymph node dissection. Pathologic outcomes are depicted in Table2. Organ confined disease (pT2) was diagnosed in

Table 1 - Characteristics of patients undergoing radical prostatectomy.

\begin{tabular}{lc}
\hline Variables & $\mathbf{N}(\%)$ \\
\hline Age median (range) & $64.0(49-74)$ \\
Race & \\
$\quad$ Caucasian & $29(57 \%)$ \\
African American & $20(39 \%)$ \\
Other & $2(4 \%)$ \\
Initial PSA & \\
0 - 4.0 & $12(23 \%)$ \\
$4.1-10.0$ & $29(57 \%)$ \\
$10.1-20.0$ & $5(10 \%)$ \\
$>20.0$ & $5(10 \%)$ \\
Gleason score on biopsy & \\
$\leq 6$ & $33(65 \%)$ \\
7 & $12(23 \%)$ \\
$8-10$ & $5(10 \%)$ \\
Microscopic disease* & $1(2 \%)$ \\
Clinical stage & \\
T1c & $38(75 \%)$ \\
T2 & $11(21 \%)$ \\
T3 & $2(4 \%)$ \\
Nerve status & \\
Bilateral nerve sparing & $15(29 \%)$ \\
Bnilateral nerve sparing & $24(47 \%)$ \\
* Focus of cancer too small to give a Gleason score. \\
$\quad 12(24 \%)$ \\
\hline
\end{tabular}

Table 2 - Pathologic characteristics.

\begin{tabular}{ll}
\hline Variables & $\mathbf{N}(\%)$ \\
\hline Pathologic stage & \\
Organ confined & $39(76 \%)$ \\
Extraprostatic extension & $10(20 \%)$ \\
Seminal Vesicle Invasion & $1(2 \%)$ \\
$\quad$ Nodal metastasis & $1(2 \%)$ \\
Gleason score of specimen & \\
$\leq 7$ & $34(67 \%)$ \\
$8-10$ & $17(33 \%)$ \\
Margin status & \\
Negative & $41(80 \%)$ \\
Positive & $10(20 \%)$ \\
Location of positive margin & \\
Apex & 2 \\
Posterolateral & 9 \\
Anterior & 0 \\
Bladder neck & 0 \\
Extent of positive margin & \\
Focal & $7(14 \%)$ \\
Extensive & $3(6 \%)$ \\
\hline
\end{tabular}

*One patient with 2 sites of positive surgical margin (apex and posterolateral)

$76 \%$ of the patients, whereas $33 \%$ of patients had poorly differentiated tumors. Positive lymph nodes (non-microscopic disease) were found in only $2 \%$ of patients. The overall positive surgical margin rate was $20 \%$ (10 patients). Seventy percent of the patients with positive surgical margins were found to have extraprostatic disease. Table- 2 depicts the location of the positive surgical margins in our series. Three patients had positive surgical margins at the bladder neck and 2 patients had persistent benign prostatic tissue at the bladder neck. These patients had wider resections of the affected bladder necks until the frozen sections were negative for cancerous or benign prostatic tissue. Then the bladder neck was reconstructed. Final pathologic evaluation of bladder neck margin was negative for tumor or persistent prostatic tissue in all 51 men.

Analyses of 6 month EPIC questionnaire responses reveal only one patient was severely incontinent and a total of $9(18 \%)$ of patients reported any form of incontinence. The one severely incontinent 
patient had a recent history of proximal urethral stricture previously managed by direct visual incision of urethral stricture. In addition $9,(18 \%)$ of patients reported their urinary function to be 'a big problem'. Of these 9 patients, only 2 patients reported urinary incontinence. The other seven patients were found to have a bladder neck contracture causing their urinary bother prior to their 6 month follow-up and were treated with transurethral incision of bladder neck contracture. The seven patients with bladder neck contracture reported resolution of their urinary bother after transurethral incision of bladder neck contracture. Only two of the patients who developed a bladder neck contracture had bladder neck resection and reconstruction $(\mathrm{p}<0.05$, Chi-square)

\section{COMMENTS}

Increased surgical volume has been associated with improved pathologic outcomes (i.e. decreases in rate of positive surgical margin) in patients undergoing RP $(9,10)$. In addition, our group has reported previously that recently graduated urologic oncologic trained surgeons can produce these same outcomes as more senior surgeons (11). Knowing patients with negative surgical margins have a more favorable biochemical disease-free survival (5), we routinely obtain intra-operative frozen sections of the bladder neck in an effort to decrease our incidence of positive surgical margins at the bladder neck. We resect as much of the bladder neck as is needed to obtain a negative surgical margin and then reconstruct the bladder neck. The resulting overall rate of positive surgical margin in our series was $20 \%$, and specifically $0 \%$ at the bladder neck margin. Though our follow-up is too short to comment on PSA-failure free survival we can extrapolate based on previous literature (5) that improve surgical margins translate into improve PSA-failure free survival.

Our initial incidence of positive bladder neck margin as assessed on intra-operative frozen section was approximately $6 \%$. We believe that others can obtain this low rate of positive surgical margins at the bladder neck through meticulous bladder neck dissection. This rate can be further decreased by obtaining intra-operative frozen sections and resecting any residual tissue. Our final positive margin status of $0 \%$ at the bladder neck is improved from other reports Table-3 $(2,3,12)$. There was no significant relationship between positive bladder neck margin and any preoperative factors, thus we found it difficult to predict preoperatively who would have a positive bladder neck margin. Furthermore, there was no relationship between the location and the number of positive biopsies and positive margin at the bladder neck (data not shown). At the time of surgery, all gross disease was resected en bloc with the prostate and seminal vesicles; subsequently, frozen sections were sent to pathology of the remaining bladder neck. Though previous researchers have commented on obtaining frozen sections from the apex $(13,14)$ and posterolateral $(15,16)$ regions, we have found that quite difficult in the presence of a true anatomic RP, since only minuscule tissue may be available at these sites for sampling. However, there is adequate tissue to sample at the bladder neck to ensure a negative margin at this location.

Table 3 - Location of positive surgical margins in contemporary series*

\begin{tabular}{lrccc}
\hline & Apex & Posterolateral & Anterior & Bladder Neck \\
\hline Obec, 1999(2) & $89(58 \%)$ & $17(11 \%)$ & $22(14 \%)$ & $25(16 \%)$ \\
Blute, 1997(3) & $253(48 \%)$ & $174(33 \%)$ & $11(2 \%)$ & $85(16 \%)$ \\
Salomon, 2003(12) & $26(43 \%)$ & $20(33 \%)$ & $0(0 \%)$ & $14(23 \%)$ \\
Bianco, 2003(21) & $49(59 \%)$ & $26(31 \%)$ & $6(7 \%)$ & $2(2 \%)$ \\
Present series & $2(20 \%)$ & $9(90 \%)$ & $0(0 \%)$ & $0(0 \%)$ \\
\hline
\end{tabular}

* may have involvement with more than one margin. 
Currently, we utilize various criteria in an effort to predict the chance of extraprostatic extension and possible positive surgical margins (17). Patients with a positive surgical margin were more likely to have a serum PSA $>15 \mathrm{ng} / \mathrm{mL}$, palpable disease, and Gleason score $\geq 7$ ( $<<0.05$, Chisquare). However, these criteria were not effective in predicting positive bladder neck margin in our cohort. There are several possible explanations for this. First, these factors may be related to positive margin by the neurovascular bundle and, thus, may not be indicative of criteria needed to assess the bladder neck. Next, our study group is quite diverse and thus criteria obtained by studying predominantly Caucasian patients may not be effective in predicting margin status in a cohort with greater percentage of African American or other races. Lastly, previous groups have documented higher rate of positive margin at the apex. Our study group may have been too small to produce a representative sample so the overall distribution of positive surgical margins may have been less than would be seen in a larger sample.

Thus, only 5 out of 51 patients in our study had a positive intraoperative bladder neck margin for either carcinoma or persistent benign prostatic tissue. The incidence of bladder neck contracture in our population was $14 \%$. The presence of bladder neck contracture did not correlate to bladder neck resection and reconstruction. All bladder neck contractures were detected prior to the patients' 6 -month postoperative follow-up and treated with transurethral incision of bladder neck contracture. At the 6-month follow-up, our overall continence rate was greater than $90 \%$, based on response to EPIC questionnaire, and $18 \%$ of patients reported urinary bother.

The concept of obtaining intra-operative bladder neck margins is not new. One of the first publications on this subject was reported by Lepor and coworkers in 1998 (18). In that report, the authors reported an $11 \%$ yield from intra-operative frozen section of the bladder neck that demonstrated persistent prostatic tissue that was resected. They concluded that intra-operative frozen sections of the bladder neck may be beneficial. More recently, Lepor \& Kaci reported their most current experience with intra-operative bladder neck biopsies
(19). In this report the authors state that due to the low yield of a positive bladder neck margin $(<1 \%)$ performing bladder neck frozen section analysis was not clinically helpful (19). However, it should be noted that this is the report of a single surgeon's experience with a very high-volume surgical practice and thus such favorable results may be due to meticulous, anatomic dissections by experienced surgeons.

The implications of obtaining frozen margins of the bladder neck can be incorporated into laparoscopic and robotic radical prostatectomy. One of the difficult steps in laparoscopic or robotic radical prostatectomy is division of the bladder neck. If our technique can be replicated during these procedures, then it may decrease the rate of positive margins for these procedures as well. Furthermore, this technique could also be utilized during the learning curve for RP (open, laparoscopic, and robotic) in hopes of reducing positive surgical margins at the bladder neck.

We clearly realize the limitations of this study. First, though it is a prospective study, the number of men assessed is small. These men assessed were the first 51 patients a recent urologic oncologist fellowship graduate completed in his practice. In addition, it would be quite informative to collect similar data from multiple surgeons performing intra-operative frozen sections of the bladder neck at the time of RP. Next, a control arm where bladder neck biopsies were not obtained may be useful. Lastly, longer follow-up that truly assesses PSAfailure free survival would be extremely beneficial in assessing the true oncologic potential of this procedure.

Bladder neck invasion is associated with an overall poor prognosis (20). Obtaining frozen sections can help identify these patients early for subsequent adjuvant trials. However, we believe there is a subset of patients that after a RP may have persistent disease at the bladder neck that is not invasive. It is in these patients that intra-operative sampling may be beneficial. Further prospective application of this treatment is required to determine its true overall impact on the care of prostate cancer patients.

\section{CONFLICT OF INTEREST}

None declared. 


\section{REFERENCES}

1. Walsh PC, Donker PJ: Impotence following radical prostatectomy: insight into etiology and prevention. J Urol. 1982; 128: 492-7.

2. Obek C, Sadek S, Lai S, Civantos F, Rubinowicz D, Soloway MS: Positive surgical margins with radical retropubic prostatectomy: anatomic site-specific pathologic analysis and impact on prognosis. Urology. 1999; 54: 682-8.

3. Blute ML, Bostwick DG, Bergstralh EJ, Slezak JM, Martin SK, Amling CL, et al.: Anatomic site-specific positive margins in organ-confined prostate cancer and its impact on outcome after radical prostatectomy. Urology. 1997; 50: 733-9.

4. Greene FL, Page DL, Fleming ID, Fritz AG, Balch CM: AJCC Cancer Staging Manual, 6th ed. New York, Springer-Verlag. 2002.

5. Babaian RJ, Troncoso P, Bhadkamkar VA, Johnston DA: Analysis of clinicopathologic factors predicting outcome after radical prostatectomy. Cancer. 2001; 91 : 1414-22.

6. Poon M, Ruckle H, Bamshad BR, Tsai C, Webster R, Lui P: Radical retropubic prostatectomy: bladder neck preservation versus reconstruction. J Urol. 2000; 163: 194-8.

7. Gleason DF: Histologic grading of prostate cancer: a perspective. Hum Pathol. 1992; 23: 273-9.

8. Amling CL, Bergstralh EJ, Blute ML, Slezak JM, Zincke $H$ : Defining prostate specific antigen progression after radical prostatectomy: what is the most appropriate cut point? J Urol. 2001; 165: 1146-51.

9. Bianco FJ Jr, Riedel ER, Begg CB, Kattan MW, Scardino PT: Variations among high volume surgeons in the rate of complications after radical prostatectomy: further evidence that technique matters. J Urol. 2005; 173: 2099-103.

10. Catalona WJ, Smith DS: Cancer recurrence and survival rates after anatomic radical retropubic prostatectomy for prostate cancer: intermediate-term results. J Urol. 1998; 160: 2428-34.

11. Rosser CJ, Kamat AM, Pendleton J, Robinson TL, Pisters LL, Swanson DA, et al.: Impact of fellowship training on pathologic outcomes and complication rates of radical prostatectomy. Cancer. 2006; 107: 549.

12. Salomon L, Anastasiadis AG, Antiphon P, Levrel O, Saint F, De La Taille A, et al.: Prognostic consequences of the location of positive surgical margins in organconfined prostate cancer. Urol Int. 2003; 70: 291-6.
13. Shah O, Melamed J, Lepor H: Analysis of apical soft tissue margins during radical retropubic prostatectomy. J Urol. 2001; 165: 1943-8; discussion 1948-9.

14. Tsuboi T, Ohori M, Kuroiwa K, Reuter VE, Kattan MW, Eastham JA, et al.: Is intraoperative frozen section analysis an efficient way to reduce positive surgical margins? Urology. 2005; 66: 1287-91.

15. Cangiano TG, Litwin MS, Naitoh J, Dorey F, deKernion JB: Intraoperative frozen section monitoring of nerve sparing radical retropubic prostatectomy. J Urol. 1999; 162: 655-8.

16. Goharderakhshan RZ, Sudilovsky D, Carroll LA, Grossfeld GD, Marn R, Carroll PR: Utility of intraoperative frozen section analysis of surgical margins in region of neurovascular bundles at radical prostatectomy. Urology. 2002; 59: 709-14.

17. Kamat AM, Jacobsohn KM, Troncoso P, Shen Y, Wen $\mathrm{S}$, Babaian RJ: Validation of criteria used to predict extraprostatic cancer extension: a tool for use in selecting patients for nerve sparing radical prostatectomy. J Urol. 2005; 174: 1262-5.

18. Lepor H, Chan S, Melamed J: The role of bladder neck biopsy in men undergoing radical retropubic prostatectomy with preservation of the bladder neck.J Urol. 1998; 160: 2435-9.

19. Lepor H, Kaci L: Role of intraoperative biopsies during radical retropubic prostatectomy. Urology. 2004; 63: 499-502.

20. Poulos CK, Koch MO, Eble JN, Daggy JK, Cheng L: Bladder neck invasion is an independent predictor of prostate-specific antigen recurrence. Cancer. 2004; 101: $1563-8$.

21. Bianco FJ, Grignon DJ, Sakr WA, Shekarriz B, Upadhyay J, Dornelles E, et al.: Radical prostatectomy with bladder neck preservation: impact of a positive margin. Eur Urol. 2003; 43: 461-6.

Accepted after revision: September 7, 2007 